\title{
Cross-modal facilitation is not specific to self-face recognition*
}

* Commentary on Platek, S.M., Thomson, J.W., Gallup, G.G. (2004). Cross-modal self recognition: The role of visual, auditory, and olfactory primes. Consciousness and Cognition, in press

Recently, Platek, Thomson and Gallup (2004) reported a series of three experiments that were aimed at determining how information about the self from different modalities and domains affects self-face recognition. Their results indicated that being exposed to one's own body odour, and seeing or hearing one's own name all facilitated the recognition of one's own face. No cross-modal facilitation was found for other familiar persons and for unfamiliar individuals. These authors claimed that this pattern of results suggests that the perceptions of self in the olfactory, auditory, and visual domains are not mutually exclusive but rather interact and provide for integrative processing of self-information. They concluded that "these priming effects on processing information about the self strongly suggest that rather than being modality specific/dependent, the sense of self is represented by a larger multi-modal phenomenon".

In their paper, Platek et al. present cross-modal facilitation of face recognition as if this phenomenon was specific to self-face recognition. This interpretation is consistent with their own results that showed no priming of other (familiar or unfamiliar) individuals' faces from their respective odour, spoken name or written name. However, cross-modal facilitation of familiar face recognition, in general, was predicted by current Interactive Activation and Competition (IAC) models of face recognition (Brédart, Valentine, Calder \& Gassi, 1995; 
Burton, Bruce \& Johnston, 1990; Burton \& Bruce, 1992; for a review see Young \& Burton, 1999) and has been empirically demonstrated in several experiments. Cross-modal priming of a target person's characteristic from another characteristic of that person occurred when the delay from the offset of the prime and the onset of the target was short. This phenomenon has been called "self priming"; in this expression "self" does not refer to the participant's identity but rather to the fact that the prime and the target share the same identity. The presentation of a person's name facilitated the recognition of this person's face (Calder \& Young, 1996), and conversely, the presentation of a person's face as a prime facilitated the recognition of that person's name (Calder \& Young, 1996: Calder, Young, Benson \& Perrett, 1996). The short interval between primes and targets $(250 \mathrm{msec})$ in the studies by Calder and collaborators was similar to that used in Platek et al.'s experiments on name to face priming (400 msec in experiment 2 and $200 \mathrm{msec}$ in experiment 3; personal communication). In previous literature, long term cross-modal facilitation of person recognition has also been reported. Indeed, results from different experiments showed that making a familiarity decision to an auditory presentation of a familiar person's name primed a familiarity decision to the same person's name presented visually (Valentine, Hollis \& Moore, 1998; Hollis \& Valentine, 2001).

From these studies it appears that cross-modal facilitation of face recognition is not exclusive to self-face recognition. The fact that such cross-modal priming was not obtained for familiar face recognition in the Platek et al. study is in contrast with previous studies. It is possible that some aspects of their procedure prevented the occurrence of the effect. For instance, the number of participants was small (from 9 to 12 per experiment), smaller than in the previous studies (usually at least 16 per experiment).

From their own results, Platek et al. take the occurrence of cross-modal facilitation as a sign of integrative processing of self-information. They implicitly oppose this integrative selfprocessing to a non-integrated (modality specific) processing of information about other 
people, including familiar individuals. However, since cross-modal facilitation is not specific to self-recognition, its occurrence cannot be seen as a piece of evidence that information about oneself is represented in a qualitatively different way from information about other familiar individuals. Platek et al.'s results may be better interpreted as a demonstration that crossmodal facilitation of self recognition can be obtained in conditions that did not yield facilitation of another familiar face recognition. These results would merely reflect the fact that the magnitude of the cross-modal facilitation may be higher for self-recognition than for the recognition of other familiar faces, even for the recognition of very familiar faces such as the mother's or a significant other's face (i.e. the kind of familiar individuals used in Platek et al.'s study).

If, as Platek et al. suggest, cross-modal priming indicates integrative processing of information from different modalities (or different stimulus domains), the fact such crossmodal facilitation in the recognition of other familiar individuals has been demonstrated in previous research is a clue that the recognition of these individuals' faces also involves this kind of integrative processing. However, because we are experts on ourselves, it is likely that this integration is more complete (in the sense that it may involve a wider range of characteristics such as name, body, odour, voice, gait, or even phone number) for selfrecognition than for the recognition of others. Some characteristics such as the name or the odour presumably yield stronger cross-modal facilitation of self-face recognition relative to the recognition of other familiar individuals. However, it is also possible that some characteristics (for instance the gait) would be more efficient in facilitating the recognition of a familiar person's face than the recognition one's own face.

Determining what is specific to self-face processing requires an evaluation of what type of information yields cross-modal or cross-domain facilitation both in self-face recognition tasks and in tasks that require the recognition of other highly familiar individuals. Such a 
comparison might lead to the conclusion that, on average, cross-modal facilitation is stronger in self-face recognition as compared with the recognition of familiar faces, or that some properties produce cross-modal facilitation in self-recognition tasks only. On the other hand, unless the results available in the face recognition literature are wrong, this comparison should not lead us to conclude that cross-modal facilitation is specific to self-face recognition.

\section{References}

Brédart, S., Valentine, T., Calder, A., \& Gassi, L. (1995). An interactive activation model of face naming. The Quarterly Journal of Experimental Psychology, 48A, 466-486.

Burton, A.M., Bruce, V., \& Johnston, R.A. (1990). Understanding face recognition with an interactive activation model. British Journal of Psychology, 81, 361-380.

Burton, A.M., \& Bruce, V. (1992). I recognize your face but I can't remember your name: A simple explanation? British Journal of Psychology, 83, 45-60.

Calder, A.J. \& Young, A.W. (1996). Self-priming: A short-term benefit of repetition. The Quarterly Journal of Experimental Psychology, 49A, 845-861.

Hollis, J., \& Valentine, T. (2001). Proper name processing: Are proper names pure referencing expressions ? Journal of Experimental Psychology: Learning, Memory, and Cognition, 27, 99-116.

Platek, S.M., Thomson, J.W., Gallup, G.G. (2004). Cross-modal self recognition: The role of visual, auditory, and olfactory primes. Consciousness and Cognition, in press

Young, A.W., \& Burton, A.M. (1999). Simulating face recognition: Implications for modelling cognition. Cognitive Neuropsychology, 16, 1-48.

Valentine, T., \& Hollis, J., \& Moore, V. (1998). On the relationship between reading, listening, and speaking: It's different for people's names. Memory \& Cognition, 26, 740-753. 
Serge Brédart

Department of Cognitive Science (B-32)

University of Liège

4000 Liège, Belgium

Email: serge.bredart@ulg.ac.be 